\title{
Hamman-Rich syndrome: a forgotten entity
}

\author{
William Newmarch1, Angelica Puopolo1, Madina Weiler1, Brian Casserly2 \\ 1 Graduate Entry Medical School, University of Limerick \\ 2 Respiratory Division, University Hospital Limerick, Ireland
}

\begin{abstract}
The following report outlines the case of a 76-year-old gentleman who presented to the hospital with acute interstitial pneumonitis, a rare and rapidly progressive type of idiopathic interstitial pneumonia. The patient initially presented with a three-week history of progressive shortness of breath and cough which was subsequently diagnosed as community acquired pneumonia. Treatment with oral antibiotics was unsuccessful resulting in re-presentation the following week with type one respiratory failure (hypoxemia without hypercapnia). Investigations revealed widespread inflammatory changes consistent with an acute inflammatory process - neutrophilia, elevated D-dimers, raised C-reactive protein (CRP) and erythrocyte sedimentation rate (ESR), as well as bilateral interstitial infiltrates on chest radiograph. Intravenous steroids, antibiotics and antiviral medications were initiated before an urgent transfer to the intensive care unit was required for intubation. An open lung biopsy, in conjunction with the clinical picture, confirmed the diagnosis of acute interstitial pneumonitis. The significance of this report is to highlight the rapid and destructive clinical course of a rare type of pneumonitis, which initially presented as a routine and innocuous diagnosis in our patient.
\end{abstract}

Corresponding author: William Newmarch, Graduate Entry Medical School, University of Limerick, 33 Mulcair Road, Raheen, Limerick, Ireland. Tel.+353.83.187-9766. E-mail: 14076926@studentmail.ul.ie

Key words: Hamman-Rich; acute interstitial pneumonitis; interstitial lung disease

Authors' contribution: WN, contributed the Discussion section and compiled the case report, in addition to gathering patient data and any relevant information regarding the case; AP, contributed the Case Report section of the manuscript; MW, contributed the Abstract and Introduction sections of the manuscript and assisted with gathering patient information; $\mathrm{BC}$, oversaw the report, including making final edits and assisting with compiling of the manuscript.

Conflict of interest: the authors declare no conflict of interest.

Received for publication: 25 November 2016

Accepted for publication: 10 April 2017

CCopyright W. Newmarch et al., 2017

Tipografia PI-ME Editrice, Italy

Monaldi Archives for Chest Disease 2017; 87:799

doi: 10.4081/monaldi.2017.799

This article is distributed under the terms of the Creative Commons Attribution Noncommercial License (by-nc 4.0) which permits any noncommercial use, distribution, and reproduction in any medium, provided the original author(s) and source are credited.

\section{Introduction}

Acute interstitial pneumonitis, also known as Hamman-Rich syndrome or acute interstitial pneumonia, was first described in 1935 by authors Hamman and Rich, physicians at Johns Hopkins, in their case report on four patients with rapidly developing fibrosis of the lungs [1]. It is characterized by:

1. acute onset of respiratory symptoms resulting in severe hypoxia or acute respiratory failure;

2. bilateral lung infiltrates on radiographic imaging;

3 . the absence of an identifiable etiology or predisposing condition despite appropriate clinical investigations, and

4. histological demonstration of diffuse alveolar damage (DAD) $[2,3]$. In this way, acute interstitial pneumonitis (AIP) is a subgroup of acute respiratory distress syndrome (ARDS), distinguished by the absence of a clinically identifiable precursor. Patients frequently present after a non-specific flu-like illness. The underlying cause of this condition, although poorly understood, is hypothesized to arise from a single insult. This is supported by the identical temporality of lung lesions in patients with AIP; a feature which suggests that an acute episode of lung injury occurred at a single point in time. This distinguishes AIP from usual interstitial pneumonia as the pathologic lesions are typically of different ages with varying areas of interstitial inflammation, normal lung parenchyma, fibroblast foci, and honeycomb change [4]. The alveolar damage in AIP occurs in three phases - an acute exudative phase, a subsequent organizing proliferative phase, and a final fibrotic (or healed) phase [3,5].

Treatment of this condition is mainly supportive, aimed at management of respiratory failure and complications. The role of steroids in treatment remains unsubstantiated [4]. Prognosis is poor, with a reported mortality rate of $70 \%$ at three months [6].

\section{Case Report}

A 76-year-old male presented to the emergency department with a three-week history of persistent productive cough and shortness of breath. After clinical assessment in the emergency department, a diagnosis of community acquired pneumonia was established. This was supported by the finding of raised inflammatory markers, specifically a raised neutrophil count of $8.15 \times 109 / \mathrm{L}$, an elevated C-reactive protein (CRP) of $128 \mathrm{mg} / \mathrm{L}$ and erythrocyte sedimentation rate (ESR) of $81 \mathrm{~mm} / \mathrm{h}$, in addition to bilateral infiltrates on imaging, markedly worse on the left side. Urea and electrolytes were in the normal ranges and blood cultures came back negative. His initial chest radiograph is shown in Figure 1. Background history was notable for diabetes mellitus, ischemic heart disease with one previous stent, atrial fibrillation with prior ablation and long-term amiodarone treatment, and a 30-pack year smoking history with no previous record of lung disease. He was prescribed a standard treatment of amoxicillin/clavulanic acid and clar- 
ithromycin for community acquired pneumonia with a CURB-65 score of 2 , before being discharged home with a respiratory outpatient appointment scheduled two-weeks later for follow-up.

No improvement was noted on antibiotic therapy and he returned to the emergency department six days later complaining of increasing dyspnoea. Laboratory investigations revealed a worsening neutrophilia as well as a markedly raised D-dimer level. Arterial blood gas analysis was significant for type one respiratory failure (oxygen saturation of 78\%), which responded to supplementary oxygenation at $2 \mathrm{~L} / \mathrm{min}$. Steroid treatment (200 mg hydrocortisone TDS, eventually increased to $1 \mathrm{~g}$ per day of methylprednisolone for three days) was subsequently initiated in addition to broad spectrum antibiotics (piperacillin/tazobactam and vancomycin). A computed tomography (CT) pulmonary angiogram showed extensive groundglass opacification throughout both lung fields and widespread ill-defined areas of consolidation with visible air bronchograms as shown in Figure 2.

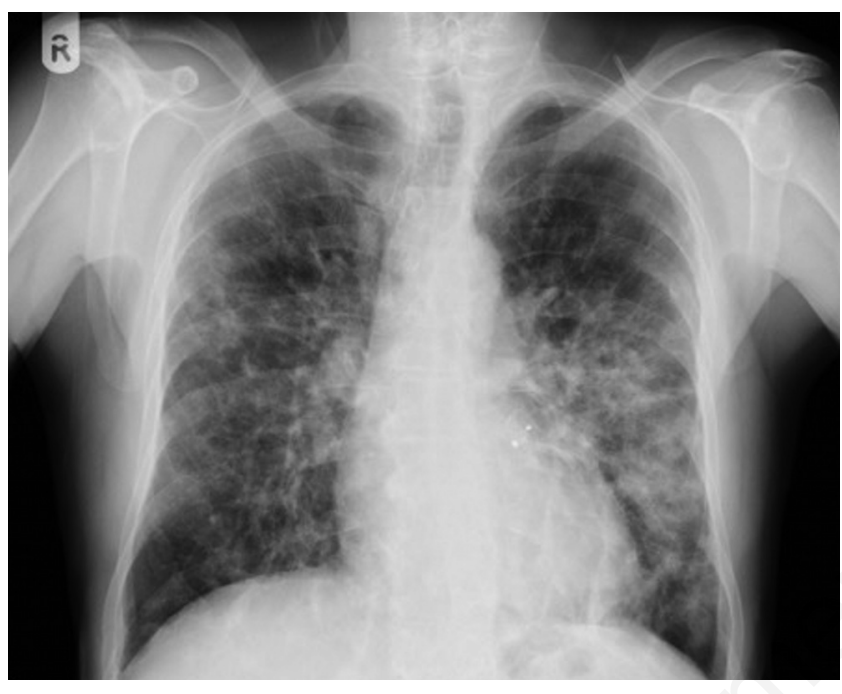

Figure 1. Initial chest radiograph notable for bilateral infiltrates most in keeping with an infective process.

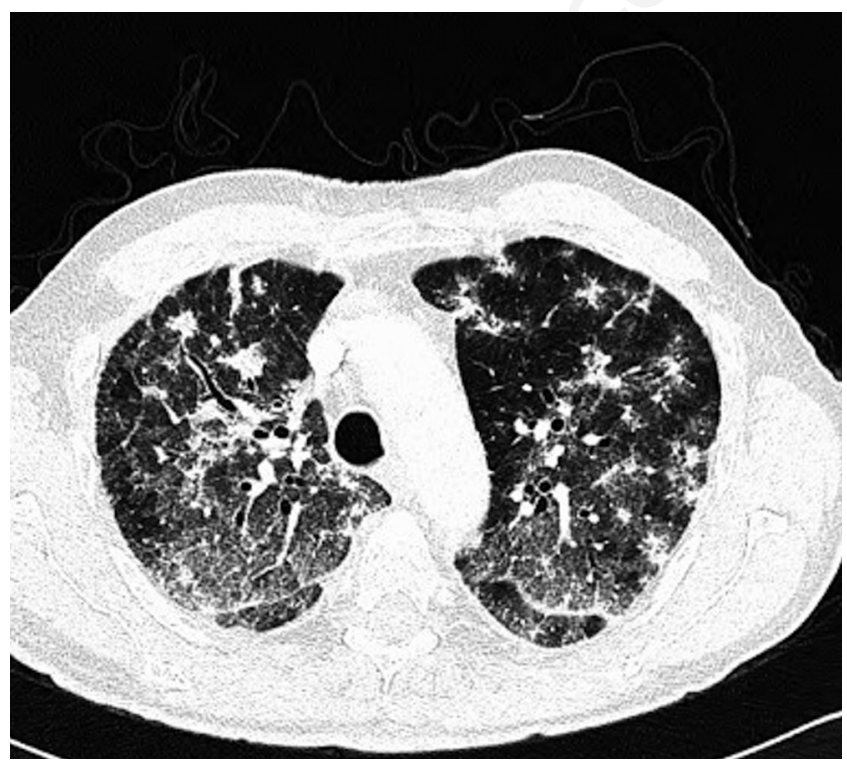

Figure 2. Computed tomography (CT) pulmonary angiogram revealing widespread ground-glass opacification and ill-defined areas of consolidation with visible air bronchograms.
No evidence of pulmonary emboli was noted. As a result, trimethoprim/sulfamethoxazole and levofloxacin were added to the treatment regime. His clinical condition continued to deteriorate overnight as evidenced by an increasing oxygen demand of $10 \mathrm{~L} / \mathrm{min}$ to maintain an oxygen saturation greater than $90 \%$.

On day two of admission, a bronchoscopy was performed which failed to show evidence of pulmonary hemorrhage or endobronchial lesions. Biopsies were negative for malignant cells, pneumocystis jiroveci or acid-fast bacilli, and cultures were only positive for commensal organisms. Histology did, however, show prominent organizing intra-alveolar fibrin with reactive type II pneumocyte hyperplasia. Post bronchoscopy, the patient required transfer to the intensive care unit (ICU) for non-invasive ventilation to maintain oxygen saturations between 88 and $90 \%$. Bedside echocardiography showed bilateral Kerley B lines but reasonable left ventricle function. Furthermore, brain natriuretic peptide (BNP) was elevated at 2023. An atypical pneumonia screen as well as human immunodeficiency virus (HIV) testing and viral swabs were all negative. Planning was then begun for transfer to a separate hospital with appropriate cardiothoracic facilities necessary for open lung biopsy.

On day three of admission the patient was intubated for transfer with an evolving ARDS picture. The ratio between the partial pressure of arterial oxygen $\left(\mathrm{PaO}_{2}\right)$ and the fraction of inspired oxygen $\left(\mathrm{FiO}_{2}\right)$, $\mathrm{PaO}_{2}: \mathrm{FiO}_{2}$, was initially 135 in ICU but deteriorated to 67 pre-transfer. The normal ratio is greater than 500 , with any value less than 200 thought to signify ARDS. A portable chest radiograph showed persistent ill-defined opacities throughout both hemithoraces as seen in Figure 3.

Transfer of the patient was arranged to the care of the cardiothoracic team where he underwent an open lung biopsy on day four of admission. Histological analysis revealed an organizing pneumonitis with mild diffuse interstitial fibrosis, and diffuse thickening of alveolar septa with reactive type II pneumocyte hyperplasia. Scattered thromboemboli and pulmonary hypertensive changes were noted as well. The histology is illustrated in Figure 4.

Amiodarone toxicity was strongly considered due to the patient's history of long-term amiodarone treatment, but these findings were more in keeping with the second phase or 'organizing phase' of Hamman-Rich. This phase classically displays features of thickened

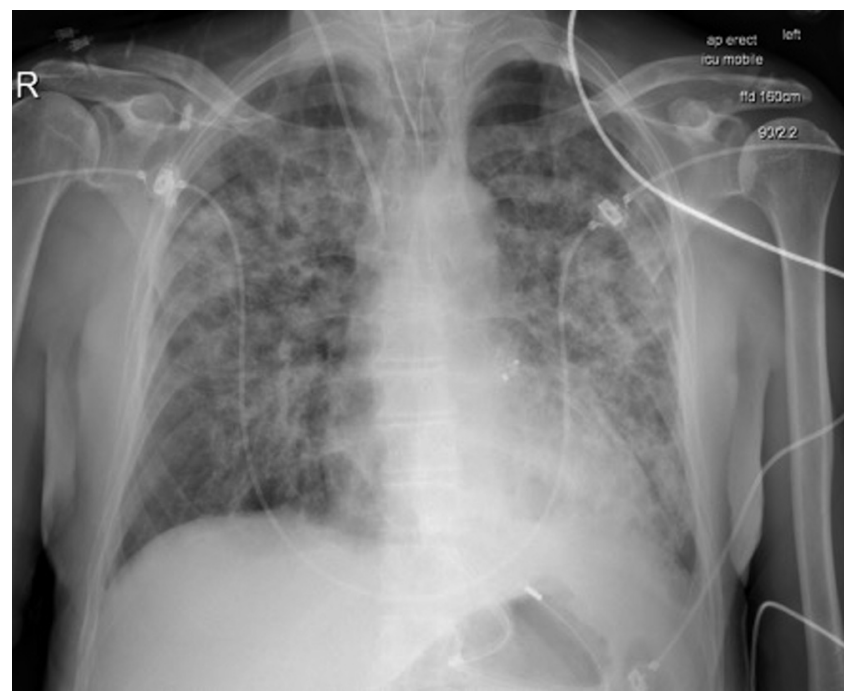

Figure 3. Portable chest radiograph showing persistent widespread opacification throughout both hemithoraces. 


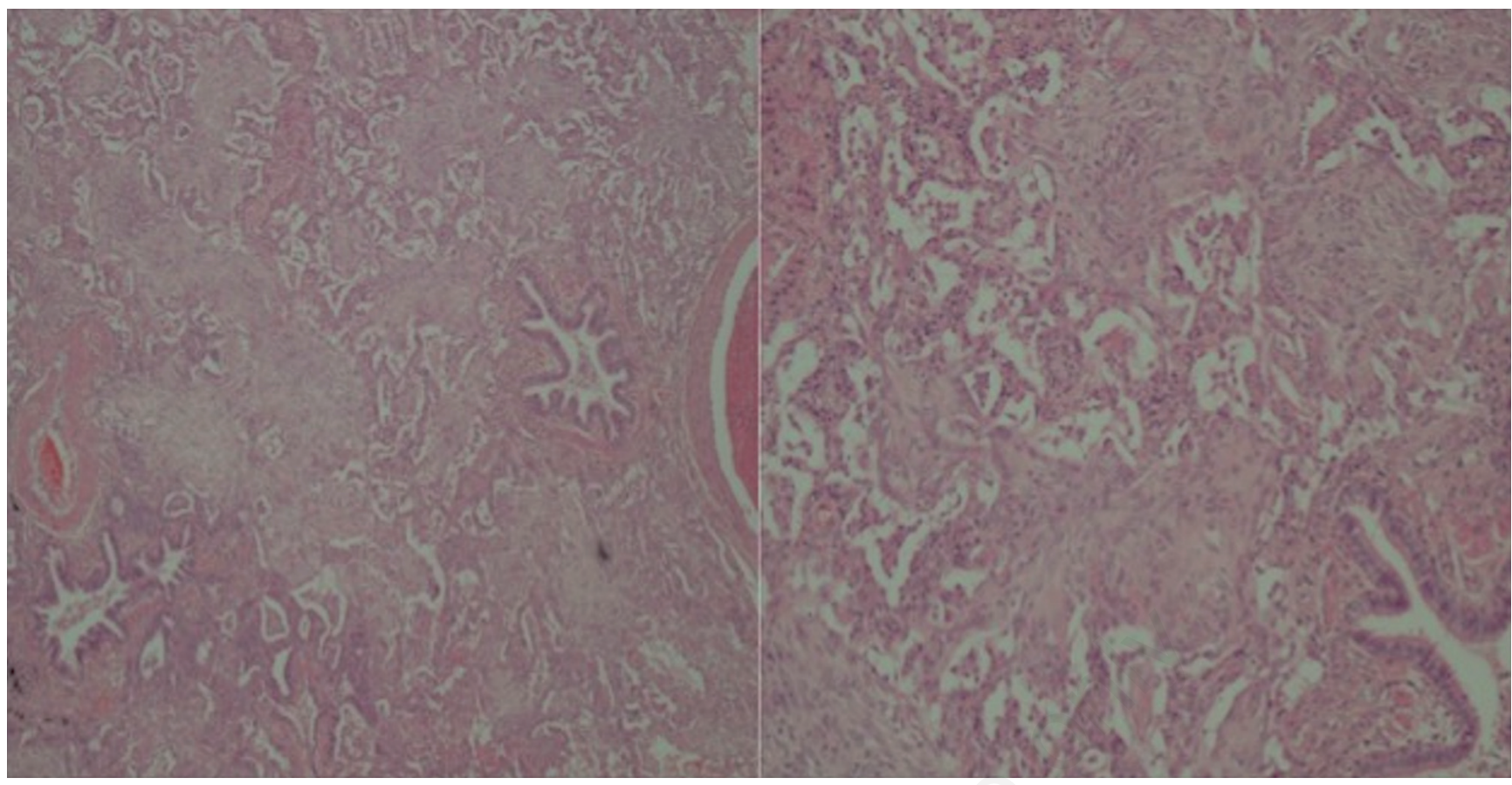

Figure 4. Lung biopsy specimen illustrating the diffuse thickening of alveolar septa with reactive type II pneumocyte hyperplasia.

alveolar septa with type II pneumocyte hyperplasia, collapse and apposition of adjacent alveolar septa, hyaline membranes in focal areas alongside alveolar septa, and thrombi in small arteries. Diffuse alveolar damage, along with the concurrent ARDS and bilateral infiltrates on chest radiograph, are the diagnostic hallmarks of Hamman-Rich syndrome. The absence of characteristic lipid-laden, 'foamy' macrophages on histology, combined with the worsening clinical picture despite steroid therapy and cessation of amiodarone treatment, helped to exclude amiodarone toxicity from the differential. Moreover, the lack of any high attenuation observed in the liver and spleen on CT imaging, typically a result of the accumulation of iodinated amiodarone in tissue macrophages, ruled out amiodarone toxicity as well.

Post open lung biopsy, a residual pneumothorax developed that required insertion of a chest drain on day five of admission. A tracheostomy was required on day seven of admission to maintain adequate ventilation. Progressively increasing oxygenation demands led to the discontinuation of hydrocortisone and the commencement of methylprednisolone in its place. Acyclovir treatment was also started initially due to a positive polymerase chain reaction (PCR) for herpes simplex virus (HSV), but a subsequent tissue culture failed to show any cytopathic effect. The HSV-1 isolated from lung secretions in mechanically ventilated patients is more often a marker, rather than a mediator, of severe illness [7].

Unfortunately, the patient continued to deteriorate in the ICU despite supportive therapy. On day 13 , a clinically distended abdomen, lactic acidosis and mottled extremities were noted. Noradrenaline was administered and concern was raised regarding the possibility of an intra-abdominal perforation in the setting of prolonged highdose steroid therapy. However, his clinical condition was deemed too unstable for transfer to imaging for further investigations. The patient was managed palliatively before expiring on day 14 of admission.

\section{Discussion}

A search for other antecedent causes of this patient's presentation was unsuccessful. Many pulmonary diseases present similarly to AIP and routine laboratory investigations are nonspecific for AIP. The primary role of diagnostic investigation, therefore, is to identify alternative diagnoses. This testing involves laboratory testing, imaging, microbiology, bronchoalveolar lavage, and lung biopsy. The differential diagnoses of AIP include: ARDS; cardiogenic pulmonary edema; exacerbation of connective tissue disorders such as dermatomyositis, polymyositis, Sjögren's syndrome, or systemic lupus erythematosus; alveolar hemorrhage; infection; drugs; or toxic inhalants [8].

Laboratory testing can be used to test for connective tissue disorders and signs of heart failure leading to cardiogenic pulmonary edema. Notably, AIP is associated with a peripheral leukocytosis of 12,100 cells/microL on average [4]. Chest imaging in AIP often appears similarly to ARDS. Microbiologic investigation of nasopharyngeal aspirates, sputum, serum, and urine are helpful in identifying influenza, pneumocystis jiroveci, and atypical pneumonias respectively. Most patients with AIP have a nonproductive cough, therefore bronchoscopy and bronchoalveolar lavage is often used to attain a respiratory tract sample [9]. Furthermore, a transbronchial biopsy obtained via flexible bronchoscopy is the preferred biopsy method when the suspected disease is predominantly centrilobular.

If minimally invasive testing does not support an alternative pathology, surgical lung biopsy may be necessary to diagnose AIP [10]. Lung biopsy can confirm diffuse alveolar damage and play a major role in excluding alternative diagnoses, however lung biopsies may pose a serious risk to the patient as well. The most common complication is an air-leak which occurs in $6-12 \%$ of cases, while the most serious complication of an open lung biopsy is death [11]. Due to the potential to either exacerbate or induce lung injury, the use of open lung biopsy as 
a diagnostic tool in the acute setting of hypoxemic respiratory failure is becoming increasingly less common. Given the benign nature of the initial presentation, it is most common to diagnose patients histologically in the organizing phase of disease progression. Our patient was biopsied via bronchoscopy on day two of admission (day seven post initial presentation and discharge) and via open lung biopsy on day four of admission - this is favorable in terms of early attempts at diagnosis when compared to other case studies available for AIP [12].

In patients with minimal symptoms, close observation with repeated pulmonary function testing is appropriate [10]. However, most patients with AIP present with hypoxemia requiring intubation and mechanical ventilation within a few days [4]. In these severe cases, open lung biopsy may be the only modality that can correctly identify a treatable disease process, and change the course of therapy [13,14]. Video assisted thoracoscopic surgery (VATS) is a less invasive surgical option compared to thoracotomy, but the requirement of one lung ventilation precludes its use in patients with severe respiratory impairment. Ultimately, patients should be assessed on a case-by-case basis. A clinical diagnosis of AIP can be made based on the clinical syndrome of idiopathic ARDS combined with the absence of alternative diagnoses after investigation. Many patients with AIP will be diagnosed using the clinical history, physical exam, and the less invasive investigative modalities listed above. In these patients, a lung biopsy will not change the course of therapy and therefore should be avoided.

Although the pathologic cascade involved in the development of AIP is not fully understood, it is clear that it is mediated, at least in part, by both pro-inflammatory and anti-inflammatory cells [6]. Other studies have suggested a possible role played by natural killer cells and chemokines in abnormal wound repair that may catalyze the transition from an initial acute lung injury to progressive fibrosis [15,16]. Further investigations into the involvement of these particular inflammatory mediators may allow for better understanding of the pathogenesis of AIP and the subsequent development of an agent with the potential to prevent disease progression [15,17].

The use of cyclophosphamide in the acute setting has been explored in the literature, but its use has been inadequately studied and is lacking in robust proven benefit as a result [17]. In addition, the diagnostic criteria for AIP, which requires out-ruling of infectious conditions with similar clinical presentations, may result in cyclophosphamide (or other immunomodulatory agents) being reserved for less rapidly progressing ARDS variants in practice. Certainly, in our patient, conclusive biopsy results were only available by day nine, at which time the underlying disease process appears to have been well established. Therefore, immunomodulation could be presumed to have had modest, if any, benefit.

One therapy not employed in this patient was extracorporeal membrane oxygenation (ECM0). In cases similar to our patient, with increasing oxygen demand presumed to be due to the non-compliance of a damaged alveolar system, ECMO may decrease a proportion of the burden and thus facilitate patient recovery. The CESAR trial showed a survival benefit when it was used in patients with severe acute respiratory failure $[4,18]$. However, this therapy is contraindicated in patients who are unlikely to revert to standard ventilatory support. In the case of our patient, the rapidly progressive nature of the clinical course may have prevented the effective use of this therapy.

Prognosis is unfavorable for those with AIP and lung transplantation appears to be the only definitive treatment as supported by the literature [4]. In the initial phase of the disease, supportive therapy may assist with recovery from the initial insult, but success varies among patients in an unpredictable fashion. Furthermore, the severity of the interstitial lung lesions does not appear to guide prognosis or predict the disease course. Mortality in AIP is associated with acute respiratory failure and its complications. As previously discussed, patients with hypoxemia often require noninvasive or invasive mechanical ventilation within a few days of presentation [4]. Clinicians often use corticosteroids in addition to ventilation, however the effectiveness of corticosteroids is controversial. Some reports show minimal response to immunosuppressive therapy while others show a positive response and better outcome [19]. When using mechanical ventilation to manage AIP, lung-protective strategies are used. These strategies include using a lower tidal volume or lower driving pressure [20]. While lung-protective strategies have shown to reduce mortality, AIP continues to be associated with a very poor prognosis. More than half of patients with AIP will die in hospital, while the majority of those who survive the initial hospitalization will die within six months $[3,6,12,19]$. Surprisingly, the patients who survive may have significant or nearly complete recovery of lung function [21]. This further highlights our incomplete understanding of the condition and our imperfect ability to manage it appropriately as a result.

This case represents a classical presentation of AIP, which developed following a non-specific prodrome. Both the histological and radiological findings supported a diagnosis of AIP. This ultimately led to a rapid disease progression most likely due to the increased refractory oxygen demand resulting primarily from reduced lung compliance.

\section{Concluding remarks}

1. This case serves to highlight the challenges clinicians face in both diagnosing and adequately treating AIP. The requirement to sufficiently rule out any identifiable etiology or predisposing condition can preclude an early diagnosis. As a diagnosis of exclusion, a very high index of suspicion is needed to guide the clinical investigations, especially during the initial stages of the disease. Therefore, AIP should be strongly considered as part of the differential diagnosis when presented with a case of rapidly progressive interstitial pneumonia.

2. For pulmonologists and cardiothoracic surgeons, this case illustrates the continued importance of surgical lung biopsy, which ultimately revealed the diagnosis of AIP. Although this did not change the prognosis for our patient, early confirmation with tissue biopsy may allow for intervention with immunomodulatory therapy to prevent disease progression. If the patient is stable, early biopsy with VATS provides a minimally invasive approach compared to thoracotomy. Surgical lung biopsy is not without its risks, however, exemplified by our patient developing a residual pneumothorax following the procedure. With the trend to decrease the number of surgical open lung biopsies performed in the acute setting of hypoxemic respiratory failure, more and more cases of AIP will go undiagnosed. Our case may represent one of the last examples of biopsy-confirmed AIP as a result. This may eventually lead to Hamman-Rich syndrome becoming a forgotten entity.

3. Despite a benign initial presentation, this case exemplifies the rapid and destructive clinical course of AIP. As a result, prognosis is poor, and lung transplantation is the only definitive treatment. ECMO may have some theoretical benefit in facilitating recovery, but more research is needed to determine its effectiveness in AIP.

\section{References}

1. Hamman L, Rich AR. Fulminating diffuse interstitial fibrosis of the lungs. Trans Am Clin Climatol Assoc 1935;51:154-63.

2. Mukhopadhyay S, Parambil JG. Acute interstitial pneumonia (AIP): relationship to Hamman-Rich syndrome, diffuse alveolar damage (DAD), and acute respiratory distress syndrome (ARDS). Semin Respir Crit Care Med 2012;33:476-85. 
3. Katzenstein AL, Myers JL, Mazur MT. Acute interstitial pneumonia. A clinicopathologic, ultrastructural, and cell kinetic study. Am J Surg Pathol 1986;10:256-67.

4. King TE, Jr. Acute interstitial pneumonia (Hamman-Rich syndrome). In: T. Post (Ed.), UpToDate. Waltham, MA. Accessed on: November 10, 2016.

5. Katzenstein AL, Mukhopadhyay S, Myers JL. Diagnosis of usual interstitial pneumonia and distinction from other fibrosing interstitial lung diseases. Hum Pathol 2008;39:1275-94.

6. Bouros D, Nicholson AC, Polychronopoulos V, du Bois RM. Acute interstitial pneumonia. Eur Respir J 2000;15:412-8.

7. van den Brink JW, Simoons-Smit AM, Beishuizen A, et al. Respiratory herpes simplex virus type 1 infection/colonisation in the critically ill: marker or mediator? J Clin Virol2004;30:68-72.

8. Parambil JG, Myers JL, Aubry MC, Ryu JH. Causes and prognosis of diffuse alveolar damage diagnosed on surgical lung biopsy. Chest 2007;132:50-7.

9. Kilinc G, Kolsuk EA. The role of bronchoalveolar lavage in diffuse parenchymal lung diseases. Curr 0pin Pulm Med 2005;11:417-21.

10. Raghu G. Interstitial lung disease: a diagnostic approach. Are CT scan and lung biopsy indicated in every patient? Am J Respir Crit Care Med 1995;151:909-14.

11. Kaarteenaho R. The current position of surgical lung biopsy in the diagnosis of idiopathic pulmonary fibrosis. Respir Res 2013;14:43.

12. Olson J, Colby TV, Elliott CG. Hamman-Rich syndrome revisited. Mayo Clin Proc 1990;65:1538-48.
13. Patel SR, Karmpaliotis D, Ayas NT, et al. The role of open-lung biopsy in ARDS. Chest. 2004;125:197-202.

14. King TE, Jr. Approach to the adult with interstitial lung disease: Diagnostic testing. In: T. Post (Ed.), UpToDate. Waltham, MA. Accessed on: March 25, 2017.

15. Okamoto M, Kato S, Oizumi K, et al. Interleukin 18 (IL-18) in synergy with IL-2 induces lethal lung injury in mice: a potential role for cytokines, chemokines, and natural killer cells in the pathogenesis of interstitial pneumonia. Blood 2002;99:1289-98.

16. Bruminhent J, Yassir S, Pippim J. Acute interstitial pneumonia (hamman-rich syndrome) as a cause of idiopathic acute respiratory distress syndrome. Case Rep Med 2011;2011:628743.

17. Schwarz MI. The acute (noninfectious) interstitial lung diseases. Compr Ther 1996;22:622-30.

18. Peek GJ, Clemens F, Elbourne D, et al. CESAR: conventional ventilatory support vs extracorporeal membrane oxygenation for severe adult respiratory failure. BMC Health Serv Res 2006;6:163.

19. Avnon LS, Pikovsky 0, Sion-Vardy N, Almog Y. Acute interstitial pneumonia-Hamman-Rich syndrome: clinical characteristics and diagnostic and therapeutic considerations. Anesth Analg 2009;108:232-7.

20. Petrucci N, De Feo C. Lung protective ventilation strategy for the acute respiratory distress syndrome. Cochrane Database Syst Rev 2013:Cd003844.

21. Vourlekis JS, Brown KK, Cool CD, et al. Acute interstitial pneumonitis. Case series and review of the literature. Medicine (Baltimore) 2000;7):369-78. 